\title{
A Circadian Clock in Neurospora: How Genes and Proteins Cooperate to Produce a Sustained, Entrainable, and Compensated Biological Oscillator with a Period of about a Day
}

\author{
J.C. Dunlap, J.J. Loros, * H.V. Colot, A. Mehra, W.J. Belden, M. Shi, \\ C.I. Hong, L.F. LaRrondo, C.L. BAKeR, C.-H. Chen, C. SCHWERDTFEGER, \\ P.D. Collopy, J.J. GAMSBY, AND R. LAMBREGHTS \\ Department of Genetics, *Department of Biochemistry, Dartmouth Medical School, \\ Hanover, New Hampshire 03755
}

\begin{abstract}
Neurospora has proven to be a tractable model system for understanding the molecular bases of circadian rhythms in eukaryotes. At the core of the circadian oscillatory system is a negative feedback loop in which two transcription factors, WC-1 and WC-2, act together to drive expression of the frq gene. WC-2 enters the promoter region of frq coincident with increases in frq expression and then exits when the cycle of transcription is over, whereas WC-1 can always be found there. FRQ promotes the phosphorylation of the WCs, thereby decreasing their activity, and phosphorylation of FRQ then leads to its turnover, allowing the cycle to reinitiate. By understanding the action of light and temperature on frq and FRQ expression, the molecular basis of circadian entrainment to environmental light and temperature cues can be understood, and recently a specific role for casein kinase 2 has been found in the mechanism underlying circadian temperature-compensation. These data promise molecular explanations for all of the canonical circadian properties of this model system, providing biochemical answers and regulatory logic that may be extended to more complex eukaryotes including humans.
\end{abstract}

\section{INTRODUCTION}

We know a great deal about how circadian clocks work in the cells of higher organisms because ideas and models for circadian oscillators developed in model systems have proven to be eminently extensible to other related living organisms. Fungi are the group of organisms most closely evolutionarily related to both animals and higher plants. It has been a while since they all diverged; the organisms that eventually gave rise to both fungi and animals diverged from plants long before Gondwanaland split up (http://www.palaeos.com/) (Dunlap 1999), and some years later (between 1 and 1.5 billion) the fungi and animals diverged (Heckman et al. 2001). However, it seems that by then some decisions had been made about how to build a clock, and these were kept probably because they worked. Indeed, both the logic and many of the molecules underlying the circadian clock in Neurospora are also found in animals. For this reason, Neurospora has proven to be a useful and durable model for understanding circadian biology. In this chapter, we briefly review the emergence of Neurospora as a salient model for clocks, how the central transcription-translation feedback loop (TTFL) works, and current views and opinions regarding how the TTFL can account for the canonical properties that make a rhythm circadian.

\section{DEFINING CHARACTERISTICS OF CIRCADIAN RHYTHMS}

Beatrice Sweeney defined a true circadian rhythm in this way: "A circadian rhythm is an oscillation in a biochemical, physiological, or behavioral function which under conditions in nature has a period of exactly 24 hours, in phase with the environmental light and darkness, but which continues to oscillate with a period of approximately but usually not exactly 24 hours" (Sweeney 1976). Thus, these rhythms are phased by the environment but are not a simple response to it; the period also exhibits the temperature, nutritional, and $\mathrm{pH}$ compensation that distinguish circadian rhythms from cell-cycle-regulated phenomena and from other approximately 24-hour metabolic or developmental rhythms whose period lengths are often quite temperature- or medium-dependent. Overall, it is these few characteristics of a rhythm - persistence under constant conditions, having a period length of about a day, resettable by brief interruptions of this constant regimen and compensated so that the period length varies only a little under different conditions of ambient temperature or nutrition - that define a rhythm as being circadian and unite it with similar rhythms found in a large number of organisms. In turn, these are the characteristics that molecular models for the clock need to be able to explain: How do the known circadian clock genes and proteins act and interact to yield a molecular cycle with these characteristics?

\section{A CIRCADIAN RHYTHM IN NEUROSPORA}

Under just the right conditions, Neurospora (like all fungi examined) can be made to express any of a multitude of discernible rhythms in growth rate, morphology, and even gene expression (Ingold 1971; Bünning 1973). These rhythms comprise a rich biology as they are probably adaptive to the organism's success, but most of them are not characterized as true circadian rhythms because they lack one or more of the defining characteristics. However, Pittendrigh et al. (1959) found a rhythm in asexual spore 
production (conidiation) that met the circadian criteria. It is likely that Neurospora would never have risen to any prominence as a circadian model had not Malcolm Sargent and colleagues tamed the system. Their initial landmark success (Sargent et al. 1966) was the identification of a novel strain (band, recently shown to be an allele of ras-1; Belden et al. 2007b) that clarified circadian output. band was shown to be so useful that it was incorporated into virtually every strain used for rhythm studies in Neurospora; by making it trivial to follow the clock, band truly spawned a field. During the next few years, the basic characteristics of the rhythm with respect to light entrainment (Sargent and Briggs 1967) and medium composition (Sargent and Kaltenborn 1972) were worked out. At $25^{\circ} \mathrm{C}$, the period length of the Neurospora circadian clock was about 22 hours, and it was temperature-compensated, varying from nearly 23 hours at $20^{\circ} \mathrm{C}$ to a bit under 21 hours at $30^{\circ} \mathrm{C}$. Likewise, it is close to the same when cultures are grown with different amounts of glucose or at different $\mathrm{pHs}$, and it lengthens by about 2 hours when grown, for instance, on histidine (Sargent and Kaltenborn 1972). Thus, the period of the cycle exhibits temperature, nutritional, and $\mathrm{pH}$ compensation. Environmental stimuli including light and temperature can reset the phase of the clock, which is by all criteria circadian in nature.

Sargent's pioneering work made the system reproducible and tractable and facilitated subsequent work by Feldman's lab on the genetics of the clock (Feldman et al. 1979). Although never prolific, Feldman's work has well stood the test of time in that over the next decade they genetically identified many of the core clock genes that have proven to be informative toward understanding the inner workings of the circadian TTFL, frq, prd-1 through 4, and chr (Feldman et al. 1979; Loros and Feldman 1986). The identification of these mutant strains allowed molecular analysis of the Neurospora circadian system to be initiated (shortly after that of Drosophila) in the mid$1980 \mathrm{~s}$; this resulted in the cloning of the clock gene frequency (frq) by 1986 (McClung et al. 1989) and the completion of targeted screens for clock-controlled genes (as well as the coining of this term) (Loros et al. 1989). Molecular manipulations of frq expression in the 1990s (Aronson et al. 1994a) proved frq to encode a central component of the core oscillator itself, rather than just a clock-controlled gene, and many additional clock mutants have since been characterized and cloned through analysis of the factors that regulate FRQ expression and abundance. Knowledge that the oscillation in frq expression was the equivalent of the operation of the circadian oscillator gave rise to studies on the effect of light on frq expression and the elucidation of the means through which fungal, and later animal, clocks are reset by light (Crosthwaite et al. 1995; Shigeyoshi et al. 1997). It was already known that two genes, $w c-1$ and $w c-2$, were required for light sensing so it was logical to see if they were needed for frq induction by light. Surprisingly, analysis of $w c-1$ and $w c-2$ mutant strains proved them to be arrhythmic as well as truly blind (Crosthwaite et al. 1997; Collett et al. 2002) and led to the discovery of the first PAS-PAS protein heterodimer as a transcriptional activator in a circadian feedback loop (Crosthwaite et al. 1997).
This type of complex was soon afterward also reported in mammals (see, e.g., Antoch et al. 1997) and flies (Allada et al. 1998; Darlington et al. 1998; Rutila et al. 1998) and is now a set piece in circadian feedback loops. A firm genetic and regulatory framework was in place by the mid- to late 1990 s, a model in which a heterodimer of WC-1 and WC-2 drove expression of frq and FRQ in turn acted to reduce the activity of its WC activators. Since this time, steady progress has been made in understanding the molecular bases for sustainability of the rhythm, period length, resetting of the circadian system by light and temperature cues, and gating of input cues. Most recently, Neurospora is proving to be a valuable system for examining the role of coupled feedback loops in clocks and for defining global features of circadian output.

Neurospora now represents a salient model system for the analysis and molecular dissection of circadian oscillatory systems. Through examination of this cellular oscillator, and comparative physiology and molecular biology with analogous animals clocks, we can begin to discern threads of similarity in oscillator design principles as well as in molecular components. In some cases, as with the casein kinases CK1 and CK2, it seems likely that the cell simply used the most common workhorse kinases for modulating circadian proteins in all systems. However, in other cases where a variety of choices were possible-for instance in the layout of the feedback loop (as a heterodimeric activator whose activity is depressed by its product), the mode of protein-protein interaction in the activator (PAS:PAS), or even the E3 ubiquitin ligase used to regulate clock protein turnover-it seems likely that the strong similarities observed between fungi and animals may signal shared evolutionary origins for circadian systems and the oscillators that underlie them.

\section{MOLECULAR BASES OF CIRCADIAN CLOCKS IN EUKARYOTIC MODELS: TRANSCRIPTION-TRANSLATION FEEDBACK LOOPS}

The Neurospora circadian system includes a genuine clock in which a molecular negative feedback loop based on transcription and translation runs its course over about a day. Between the period in the 1980s when the first clock genes were being cloned and mid-1990s when the basic layout of the feedback loops became clear, circadian cycles were generally viewed in much the same way as we view the cell cycle, where a series of events happen one after the other over the course of a day. Such a cycle is quite distinct from the way we view the clock cycle now, as a single step loop where the long time constant can be described by the length of time it takes to turn on gene(s) (frq, or per and tim in flies, or the pers and crys in mammals) plus the length of time it takes the protein products of these genes (FRQ, PER, or PERs and Crys) to turn off their activators (respectively, WC-1/WC-2, Cyc/Clk, or BMAL1/CLOCK). Figure 1 compares the basic layout of the circadian feedback loops in Drosophila, Neurospora, and mammals (the core feedback loops are shaded).

A brief aside is warranted here to consider whether the core TTFL described above is indeed the core of the clock 

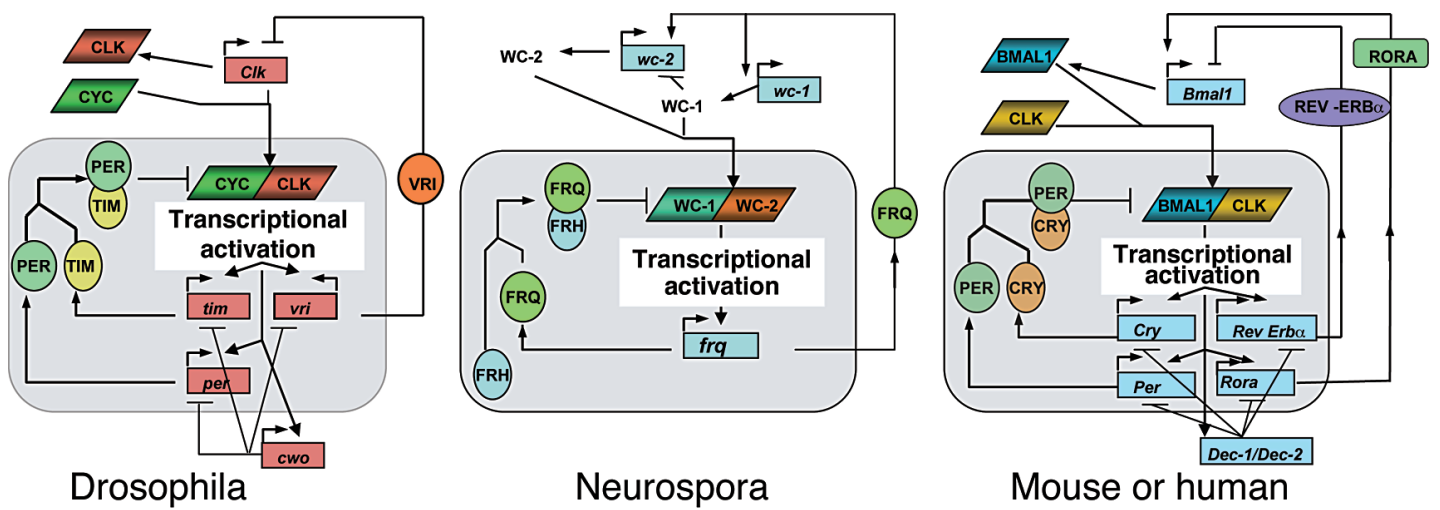

Figure 1. Similar regulatory relationships constitute the circadian oscillators in model organisms. The shaded region in the center of each loop covers the core transcription-translation feedback loop that underlies circadian rhythms. Regulatory relationships outside this core are helpful but not essential for circadian rhythmicity. (Adapted, with permission, from Bell-Pedersen et al. 2005 [C Nature Publishing Group].)

or just a reflection of output. This possibility is raised by the recent data from Synechococcus showing that a compensated circadian cycle of phosphorylation can be reconstituted in vitro from just the KaiA, KaiB, and KaiC proteins (Nakajima et al. 2005). Extensive work from the premolecular era in the late 1970s and early 1980s showed conclusively that daily timed protein synthesis was needed for operation of the clock, even in anucleate Acetabularia. In Neurospora, no level of constant frq expression has been found to be capable of supporting rhythmicity. Although carefully constructed scenarios might be mounted to question the obvious conclusion from these data (that this daily cycle is essential for the clock), in the real world, it seems undeniable that the TTFL does occur and that it drives whatever more fundamental loop(s) might exist. As explained above and below, the TTFL sets both period length and phase, and all existing molecular explanations for all the canonical circadian properties in all eukaryotes derive from it. Although considering alternative models is a useful academic exercise that may also shed light on the evolutionary origin of rhythmicity, TTFLs remain the best and only available option at this time for understanding how clocks work in cells in nature.

\section{OVERVIEW OF THE NEUROSPORA CLOCK: HOW THE MOLECULAR ELEMENTS IN THE CIRCADIAN OSCILLATOR KEEP TIME}

There are just a few components in the Neurospora cycle whose roles are well understood and several more whose actions are needed but are as yet poorly understood. The proteins WHITE COLLAR-1 (WC-1) and WHITE COLLAR-2 (WC-2) act together as a transcription factor (the WCC) to drive expression of the gene encoding another protein, FREQUENCY (FRQ); once made, FRQ acts to turn down the activity of WC-1 and WC-2. A series of kinases (CK1, Gorl et al. 2001; CK2, Yang et al. 2002; and PRD-4/checkpoint kinase 2, Pregueiro et al. 2006) and phosphatases (PP1 and PP2A, Heintzen and Liu 2007) regulate FRQ stability as well as
WCC activity and stability. Phosphorylation of FRQ makes it attractive to FWD-1, the substrate-recruiting subunit of an SCF-type E3 ubiquitin ligase (He et al. 2003); FWD-1 is the ortholog of the Slimb protein that performs a similar function in the Drosophila clock (Ko et al. 2002). Ubiquitinated FRQ is degraded in the proteasome. Indeed, once the daily cycle of FRQ synthesis and turnover was known (Garceau et al. 1997), roles for proteins such as these were anticipated. An unexpected entrant to the list of proteins in necessary supporting roles is the FRQ-interacting RNA helicase FRH, a protein that copurifies with FRQ (Cheng et al. 2005) and is essential for the cell as well as for the clock. These various molecular components interact over time in the cell, providing a glimpse of the molecular basis of subjective time.

\section{The frq Gene and Regulation of Its Expression}

frq was the second clock gene identified (Feldman et al. 1979) and the second to be cloned (McClung et al. 1989). The synthesis and processing of the frq transcripts are themselves of interest because frq has one of the most complex expression patterns of any gene known in microbes.

By the late subjective night when most of the FRQ protein in the cell has become unstable and is being degraded, WC-1 and WC-2 act together at a specific sequence in the frq promoter (the Clock Box, Froehlich et al. 2003) to drive transcription (Fig. 2). The WC proteins also bind to a second site, the proximal light regulatory element (PLRE), to mediate the light induction of frq (Froehlich et al. 2002) that serves to entrain the clock as described below. As the names imply, the Clock Box is the cis-acting site that is essential for rhythmicity, whereas the PLRE mediates most of the light induction, although the Clock Box (distal LRE) contributes to this (Froehlich et al. 2002).

Chromatin immunoprecipitation (ChIP) studies (Belden et al. 2007a) are consistent with this view, but they have also turned up some surprising regulation. WC-1 has always been shown to exist with WC-2 in solution as the White Collar Complex, so the assumption when ChIP 


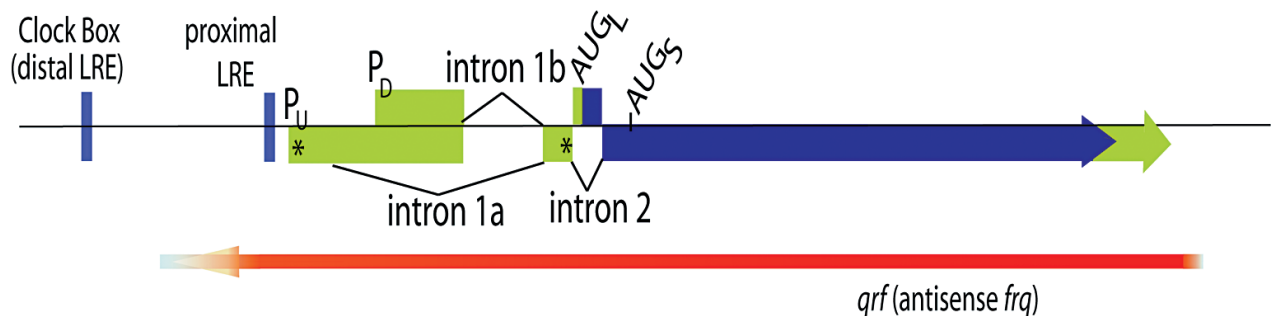

$1 \mathrm{~kb}$

Figure 2. Complex regulation and splicing involved in the expression of frq. The Clock Box and PLRE are regulatory elements upstream of $\mathrm{P}_{\mathrm{U}}$ and $\mathrm{P}_{\mathrm{D}}$, the upstream and downstream promoters, respectively. (Green) Parts of the primary transcript that are retained after splicing; (asterisks) upstream ORFs that influence the amount of FRQ made; (purple) FRQ ORF. Temperature-regulated splicing of intron 2 governs whether $\mathrm{AUG}_{\mathrm{L}}$ or $\mathrm{AUG}_{\mathrm{S}}$ is used to initiate FRQ. (Red) qrf, the antisense transcript (see text for details). (Adapted, with permission, from Dunlap 2006 [C ASBMB].)

experiments were undertaken was that WC-1 and WC-2 would be entering and leaving the Clock Box as a unit at clock phases associated with increasing and decreasing frq expression. Instead, WC-1 can always be found at both the PLRE and the Clock Box, and treatment with light results in only a minor increase in binding. In contrast to this, binding of WC-2 is highly regulated: In the dark, there is a high-amplitude cycle of WC-2 binding to the Clock Box, with binding beginning to rise in the mid-subjective night and peaking around subjective dawn, a timing consistent with the activation of frq expression. Similarly, short light treatments dramatically increase the binding of WC-2 to the PLRE and are associated with a decrease of histone H3 acetylation at the transcription start site. Not surprisingly, this regulated expression of frq is associated with remodeling of the chromatin in this area. Light elicits a very clear opening up of the chromatin at the transcription start site as assessed by micrococcal nuclease digestion, and there is an apparent but low-amplitude cycle of nucleosome remodeling at the Clock Box with, as might be expected, the peak of occupancy at the trough of frq expression and the low point at the peak of frq expression around subjective dawn.

To ascertain the means through which these changes were effected, all of the 19 homologs to yeast Swi2/Snf2like ATP-dependent chromatin remodeling enzymes have been disrupted using new methods for high-efficiency gene replacements (Colot et al. 2006), and two enzymes were found to be important for operation of the clock. One is a homolog of the yeast Fun30, mouse Etll, and human SMARCAD genes; Fun30p is known to remodel chromatin in vitro (C. Wu, pers. comm.), but its function(s) in the cell is still largely unknown. Because of its role in circadian regulation, this first gene was called clockswitch-1 (csw-1). The second is the homolog of the mammalian CHD2 and yeast Chd1 genes (W.J. Belden, unpubl.). For the $c s w-1$ knockout, there is a rhythm of frq expression but this rhythm is not overtly circadian and degrades as time goes on. It is not clear, based on the banding rhythm, whether the strains are completely arrhythmic. The loss of amplitude is the result of a failure of WC-2 to exit the Clock Box, such that the levels of frq and FRQ expression never decline to baseline but remain somewhat elevated. There appears to be no defect in the strong increase in WC-2 binding to the PLRE as a result of light exposure.
For the chd-2 knockout, rhythms in frq expression are seen, although overall, frq is at higher levels and is seen at subjective dusk, a time when frq is not normally present. Taken together, these data provide the first picture of the events happening at the frq promoter. At this point, the simplest interpretation is that these daily CSW-1 and CHD-2-mediated events assist in the chromatin remodeling that is important for the correctly timed daily expression of frq, which is in turn essential for overt rhythmicity to appear.

As a result of all this regulation at the promoter, the frq transcripts begin to appear late in the subjective night. Sense frq RNAs (Fig. 2, in green) arise from two distinct start sites $\left(\mathrm{P}_{\mathrm{U}}\right.$ and $\left.\mathrm{P}_{\mathrm{D}}\right)$ (Froehlich et al. 2002, 2003; Colot et al. 2005). A first intron can be spliced using one of two 5' donor sites ( $1 \mathrm{a}$ and $1 \mathrm{~b}$ ) with the same $3^{\prime}$ acceptor, and the second intron is alternatively spliced in a temperaturedependent manner, altogether giving rise to six major identifiable transcripts whose abundance reflects environmental conditions (Colot et al. 2005; Diernfellner et al. 2005). The alternatively spliced second intron contains the first AUG codon of the FRQ ORF ( $\mathrm{AUG}_{\mathrm{L}}$ ), so if intron 2 is removed, FRQ begins from $\mathrm{AUG}_{\mathrm{S}}$. The result is that this temperatureinfluenced splicing determines the mix of short and long FRQ proteins (purple) (Garceau et al. 1997; Liu et al. 1997; Colot et al. 2005; Diernfellner et al. 2005).

The amount of each protein also varies with temperature (Liu et al. 1997) as a result of the action not only of splicing, but also of two upstream open reading frames (uORFs). The original unspliced transcript would contain a number of these (Garceau 1996) but most are removed with intron 1; however, even after splicing, one remains at the very $5^{\prime}$ end of all transcripts along with a second one just upstream of the $5^{\prime}$ splice junction of intron 2 (Colot et al. 2005). A function of uORfs in regulating FRQ translation was predicted by Liu et al. (1997) and later confirmed by reverse genetics (Diernfellner et al. 2005). Both long and short FRQ are needed for the best robust rhythmicity, but at temperatures of less than $22^{\circ} \mathrm{C}$, more short FRQ is present and less overall FRQ is needed, whereas above $26^{\circ} \mathrm{C}$, higher overall levels of predominantly large FRQ are used (Liu et al. 1997). Despite this regulation, distinct molecular activities and/or functions for the FRQ isoforms are not yet described; 1FRQ and sFRQ appear to have indistinguishable stabilities (Liu et al. 1997) and 


\section{A CIRCADIAN CLOCK IN NEUROSPORA}

both support a functional if not entirely wild-type clock if they are present in sufficient amounts. An earlier report (Diernfellner et al. 2005) suggesting that the temperaturemodulated ratio of 1FRQ and sFRQ had a role in determining temperature-compensation has not held up (Diernfellner et al. 2007; see below); instead, this complicated temperature regulation of forms and amounts seems to help in keeping the phase of the rhythm steady across a temperature range (Dunlap and Loros 2006). As described more fully below, compensation appears to derive from a balancing of synthesis and turnover of clock components, especially FRQ (Ruoff et al. 2005), and is strongly influenced by levels of certain kinases.

A long antisense frq transcript ( $q r f$, for frq backward; red in Fig. 2) is also rhythmically expressed at low levels. This transcript shows peak expression at a phase opposite to that of frq and additionally is light induced, but the molecular basis of its activities is completely unknown. Interestingly, it does not appear to encode a protein but does appear to have a role in ensuring precise entrainment to light/dark cues (Kramer et al. 2003).

\section{What Does FRQ Do and with What Other Proteins Does It Act?}

Within a few hours of the appearance of its message in the late subjective night/early subjective morning (around point A in Fig. 3), FRQ is translated (Garceau et al. 1997). The proteins dimerize (Heintzen and Liu 2007), move to the nucleus (Luo et al. 1998), and interact with the RNA helicase FRH (Cheng et al. 2005). All of the FRQ in the cell appears to be complexed with $\mathrm{FRH}$, although the reverse is not true; FRH appears to have many roles in the cell, and a substantial pool of FRH without FRQ exists. FRH is an essential protein, a member of the SKI2 subfamily of DEAD-box-containing RNA helicase proteins. It is highly similar to yeast Mtr4p (BLASTP e-209), which is part of a yeast nuclear polyadenylation complex (LaCava et al. 2005) and a cofactor of the exosome, a complex of $3^{\prime}-5^{\prime}$ exonucleases that have a role in $3^{\prime}$-end processing, RNA maturation, and quality control; Mtr4p is also involved in mRNA export. The only published mutant of frh is an engineered partial loss-of-function strain in which expression was reduced by coexpression of a hairpin RNA (Cheng et al. 2005), so it has not yet been possible to genetically separate its functions. Interaction studies using engineered domain deletions of FRQ suggest that FRH mediates FRQ-WCC interactions, but how this might play into a role with the exosome and/or poly(A) addition or 3'-end formation can only be guessed.

It is in the nucleus where FRQ fulfills its first and major role in the clock. Specific interactions occur between the FRQ/FRH complex and the WCC (before point B in Fig. 3) (Denault et al. 2001; Merrow et al. 2001; Cheng et al. 2005). The original observations of Crosthwaite et al. (1997) were that both WC-1 and WC-2 were required for expression of frq, and this gave rise to the model wherein FRQ would act to reduce their ability to activate frq transcription (Crosthwaite et al. 1997; Froehlich et al. 2003). This is now understood to happen through the ability of FRQ to induce or promote the phosphorylation of WC-1 and WC-2 (He and Liu 2005; Schafmeier et al. 2005; Heintzen and Liu 2007). The recent detailed analysis of the events at the frq promoter using the ChIP analysis cited above, showing that WC-1 is always bound to DNA,

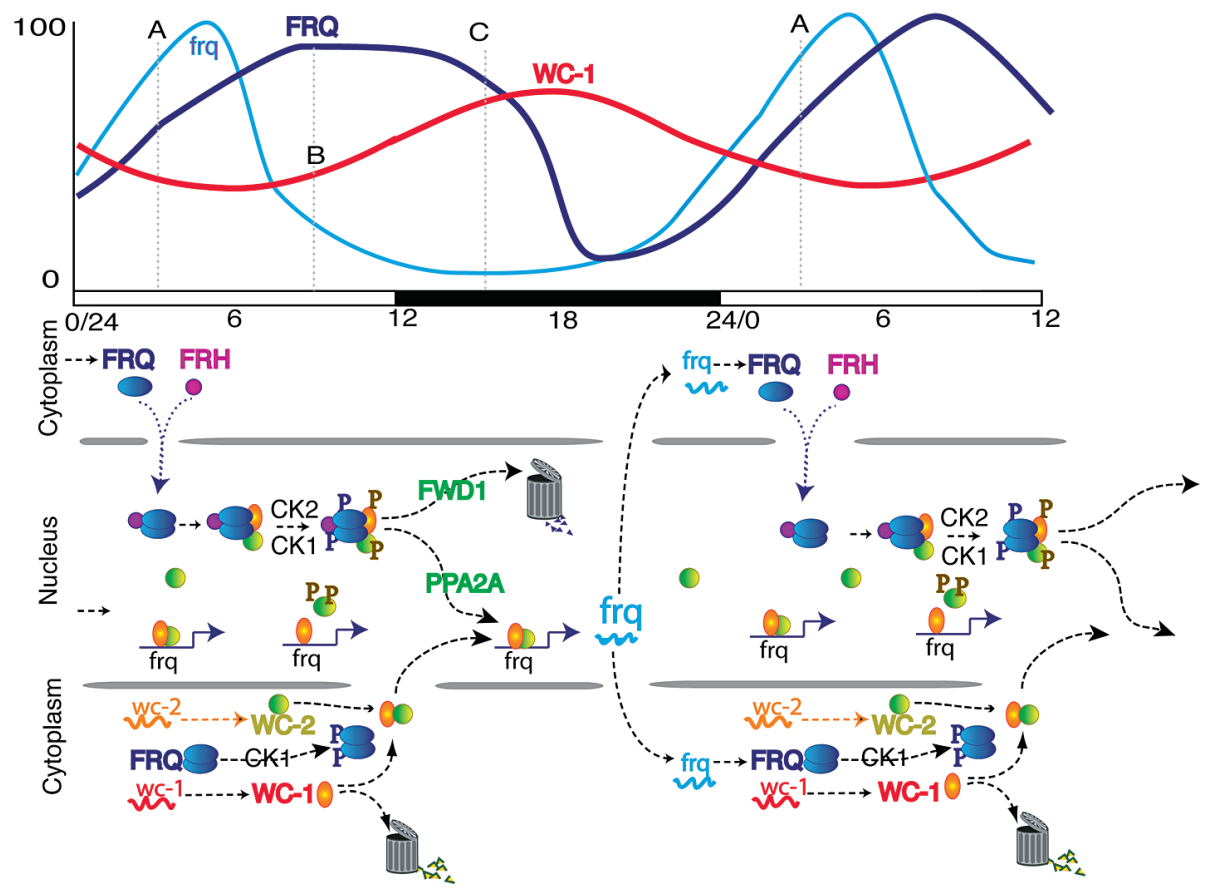

Figure 3. Molecular details within the Neurospora circadian mechanism. (Top) Cycles in the levels of clock-pertinent RNAs and proteins. (Bottom) Locations of RNAs and proteins important for the circadian oscillator are shown as a function of time, from left to right, beginning at subjective dawn (CT 0). The trash can indicates the proteasome. (Adapted, with permission, from Dunlap 2006 [C ASBMB].) 
whereas WC-2 presence is profoundly rhythmic (Belden et al. 2007a), can be seen in this light as the direct result of this cyclic phosphorylation. Both CK1 and CK2 appear to be important for this, because mutants that reduce either their activities or amounts result in enhanced binding of the WCC to the frq promoter DNA that, moreover, cannot be reduced simply through overexpression of FRQ (Heintzen and Liu 2007). By mid-subjective day, the FRQ/FRH-promoted phosphorylation reduces WCC activity and WC-2 binding to their lowest levels (Lee et al. 2000; Froehlich et al. 2003; He and Liu 2005; Belden et al. 2007a). This results in dampened expression of frq RNA such that its level begins to fall. FRQ synthesis continues as long as frq mRNA is present, so the peak in FRQ levels is broad and occurs near the end of the subjective day and into the early night.

As soon as FRQ appears, it is also phosphorylated, giving rise to multiple phosphorylation isoforms and driving its eventual turnover (Garceau et al. 1997). Several kinases are known to be involved-CK1, CK2, CAMK1, and PRD-4 (when activated by DNA damage) - and they determine the stability of FRQ (Liu et al. 2000; Gorl et al. 2001; Yang et al. 2002; Pregueiro et al. 2006). Phosphorylation of FRQ has a central role in determining the length of the circadian cycle (Liu et al. 2000), because it is this phosphorylation, or series of progressive phosphorylations, that facilitates the interaction of FRQ with the SCF-type ubiquitin ligase FWD-1. This interaction, in turn, targets FRQ for turnover in the nucleosome (around point $\mathrm{C}$ in Fig. 3); FWD-1 is later recycled through the action of the COP9 signalosome (Heintzen and Liu 2007). FRQ phosphorylation may also influence FRQ/WCC interactions (Heintzen and Liu 2007). It is now clear that environmentally induced changes in phosphorylation can affect period and also reset the clock (see, e.g., Pregueiro et al. 2006) as described more fully below.

Although the dominant role of FRQ is its exclusively nuclear action in depressing its own synthesis, a third function of FRQ relies on its continued presence in the cytoplasm as well as on its phosphorylation. Late in the subjective day, at a point probably triggered by previous phosphorylations, FRQ becomes phosphorylated at serines 885 and 887 , and this allows it to promote accumulation of WC-1 (Schafmeier et al. 2006). WC-1 is stabilized by its interaction with WC-2, so it may be that appropriately phosphorylated FRQ fosters the assembly of the WCC. In this way, the low-amplitude (and apparently dispensable) rhythm in WC-1 (Lee et al. 2000; Heintzen and Liu 2007) arises from a relatively invariant pool of spliced $w c-1$ mRNA. Additional mechanisms must contribute to this low-amplitude WC-1 rhythm, however, because it is still seen in frq-null strains (dePaula et al. 2006). Finally, FRQ also promotes the expression of $w c-2$ mRNA (Heintzen and Liu 2007), although WC-2 levels are constitutively high in any case (Denault et al. 2001).

The end result of all of these actions of FRQ is the stable and robust cycle illustrated in Figures 1 and 3: Early action results in negative feedback and late action promotes the appearance of both WC-1 and WC-2 so that WCC is maintained at an elevated level but is moreover held inactive through the action of FRQ in promoting the phosphorylation of the WCC by CK1 and CK2 and perhaps in promoting turnover of WC-2 on the frq promoter. Eventually, the precipitous phosphorylation-mediated turnover of FRQ (point C, Fig. 3) releases the WCC; it can then be recycled to an active state through the action of protein phosphatases or replaced by newly synthesized and assembled WCC, and it can reinitiate the transcription of frq mRNA in the next cycle.

Knowledge of this feedback loop goes a long way toward explaining one of the central tenets of the circadian clock: its very long time constant. The long period of the circadian day is largely accounted for by the long time it takes for FRQ to be phosphorylated and turned over. Moreover, this process clearly requires the action of more than one kinase, suggesting the possibility that they must work in tandem or sequentially in such a way that they further delay each other's actions. Interestingly, the kinetics of FRQ synthesis would appear to have relatively little to do with the length of the circadian day. As an aside, this view of the circadian cycle as a FRQ cycle provides some conceptual problems for output. In the late 1980s, early 1990s view of the clock as a series of sequential actions, it was easy to see how different activities timed to different phases of the clock cycle could be regulated. Although it was not appreciated at the time, this is now understood as the way in which different cyclins act to produce cell-cycle-stage-specific activities. However, such phase determination is not possible with the clock as a single long feedback loop. It may be that ancillary and slave oscillators have been so widely used in circadian systems to circumvent just this problem and thereby to allow accurate control of activities at a variety of circadian phases.

\section{MOLECULAR EXPLANATIONS FOR ENTRAINMENT}

Entrainment describes the process by which the phase of the clock is adjusted in response to time-of-day-specific environmental cues so that the day phase of the circadian cycle coincides with the day phase of the external world and the night phase with the night. Neurospora was the first system in which mechanisms for entrainment by light (Crosthwaite et al. 1995) and by temperature (Liu et al. 1998) were described, and the descriptions for each fell directly out of the knowledge of how the circadian feedback loop operates. Likewise, once the resetting response was understood, the means by which the clock could impact resetting to provide circadian gating could be investigated, and this was also first done in Neurospora (Heintzen et al. 2001). Consistent with the fact that the overall logic behind the feedback loop in fungi is similar to that in mammals, aspects of the light response appear to be conserved in mammals (Shigeyoshi et al. 1997). At present, too little is known about temperature responses in mammalian cells in culture to understand whether the mechanism underlying temperature resetting in cellular rhythms is also conserved. For a description of resetting by light, the nature of the photoreceptor, the mechanism of entrainment, and a clue to the mechanism of gating are each considered separately. 
In Addition to Its Role in the Clock, WC-1 is the Blue Light Photoreceptor

Most light responses described in Neurospora are specific to blue light and no red or far-red responses, such as those seen in other fungi and higher plants, have been seen. Blue-light-induced phenotypes are blocked in mutants of either white collar-1 (wc-1) or white collar-2 (wc-2) (Ballario and Macino 1997), suggesting that WC-1 and WC-2 are the principal if not the sole components for blue light responses in Neurospora. This is perhaps surprising based on genomic data that have revealed the presence of two phytochromes (Froehlich et al. 2005), one of which is circadianly regulated, as well as a strongly light-induced cryptochrome gene (Froehlich 2002); however, lightrelated phenotypes for these genes remain lacking.

Light induces mycelial carotenogenesis, resulting in the characteristic yellow-orange color of Neurospora. Mutations in genes affecting this light induction (wc-1 and $w c-2$ ) result in white mycelia and were among the first mutations described in this organism. It was hypothesized early on that these genes encoded photoreceptors (Harding and Shropshire 1980), but it was not possible to make a distinction between photoreceptors and those proteins required to transduce the signal. Macino and colleagues (Ballario et al. 1996; Linden and Macino 1997) cloned the genes and they and other investigators described the protein interactions in vitro and in vivo (Ballario et al. 1998; Denault et al. 2001; Heintzen and Liu 2007). On the basis of the fact that the WC proteins strongly resembled transcriptional activators, Macino and colleagues proposed that $w c-1$ and $w c-2$ encoded the transcription factors mediating light-induced gene expression, and that WC-1 itself might be the photoreceptor.

Macino's guess was correct: WC-1 is the protein that perceives the light signal and initiates the photoresponse (Froehlich et al. 2002; He et al. 2002). The chromophore that actually absorbs the light is FAD, and it is bound into a specialized PAS domain called a LOV (for light, oxygen, and voltage sensing) domain. Studies of other LOVdomain-containing photoreceptors including those of fungi (Crosson and Moffat 2001; Zoltowski et al. 2007) suggest that absorption of blue light causes FAD to undergo a transient covalent interaction with WC-1 inducing a conformational change in the protein. Light causes the formation of multimers of the WC proteins and enhanced transcriptional activation of WCC-bound lightresponsive promoters (Froehlich 2002). Froehlich et al. (2002) showed such a light-induced change in DNA binding of the WCC, using in-vitro-transcribed and -translated extracts primed only with $w c-1$ and $w c-2$ and dependent only on addition of FAD, indicating that these three components are sufficient for light-regulated DNA binding of these transcriptional activators.

\section{How Light Resets the Clock}

For entrainment to work, light must delay the clock into the previous day when seen early in the night and advance the clock into the next day when seen late at night. Thus, the molecular basis of resetting by light requires that the same photic cue have opposite effects on the timing mechanism depending on when in the cycle light is perceived (Crosthwaite et al. 1995). As noted above, frq mRNA peaks in the morning and this defines molecular morning (Aronson et al. 1994b; Crosthwaite et al. 1995). As a result, actions that increase frq to peak levels will tend to reset the clock to morning regardless of when they occur. Light acts rapidly through the WCC bound to the two light-response elements (LREs) within the frq promoter to induce frq (Crosthwaite et al. 1995, 1997; Froehlich et al. 2002). A plausible model for resetting (Fig. 4) works in this way: Through the subjective evening and early night, when frq is falling, light induction of frq rapidly sends the clock back toward the time of peak levels (morning) yielding a phase-delay, whereas in the late night when frq mRNA levels are rising to a midmorning peak, rapid induction of frq by light to its peak advances the clock to a point corresponding to morning (Crosthwaite et al. 1995). This remarkably simple mechanism provides a simple explanation for how resetting works: It is the same molecular mechanism-light induction of a clock component - and it is the dynamics of the feedback loop itself that causes this action to be interpreted as an advance or a delay. This is also in large part how mammalian clocks are reset by light (Shigeyoshi et al. 1997). On reflection, it is clear that the feedback loop could also be reset through light-triggered decay of a clock component that peaks at night; because per peak expression occurs at night, we correctly predicted (Crosthwaite et al. 1995) that the Drosophila clock would be reset in this way, as is the case (see, e.g., Hunter-Ensor et al. 1996).
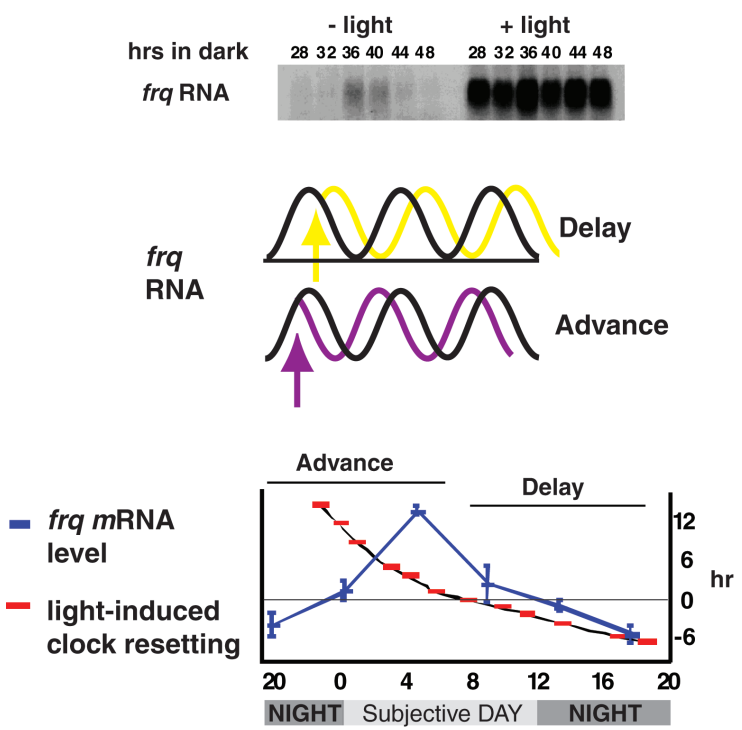

Figure 4. How light resets the clock. (Top) Cycle in frq RNA abundance and effect of brief exposure to light at any time. (Middle) Black represents the cycle of frq transcription; colored lines show the effect of transient light induction of frq on the steady-state rhythm. (Bottom) The cycle in frq transcript levels juxtaposed with response of the clock to light, showing how light seen while $f r q$ is rising leads to advances, and light seen while frq levels are falling leads to delays (see text for details). (Adapted, with permission, from Dunlap 1999 [@ Elsevier].) 
Although the model implies a wonderful simplicity in the responses to light, this may be misleading because the total light response is not just transcriptional. There is gating, as described below, but beyond this is the fact that FRQ accumulates and is phosphorylated in the light but is not significantly degraded until transfer to dark (Collett et al. 2002; Tan et al. 2004). Also in the dark, frq and FRQ synthesis reflects the feedback loop: If after transfer to darkness, there is not enough FRQ to complete the negative feedback loop, still more FRQ will be made. However, if enough FRQ is present, it will immediately begin to be phosphorylated and to progress toward degradation. Thus, depending on the duration of light treatment before darkness, the synthesis/decay kinetics of FRQ will vary substantially and the time separating frq transcription and the peak of FRQ can change by many hours. Additionally, $f r q$ is acutely induced by light and FRQ does not block this light induction (Crosthwaite et al. 1995). Furthermore, FRQ is phosphorylated in a complex manner as described above, some of it regulating turnover and some influencing activity or interactions with other proteins (see, e.g., Liu et al. 2000; Heintzen and Liu 2007), so depending on how long a strain has been in light, the FRQ present is qualitatively different. Finally, again as described, a light-inducible antisense frq message is expressed out-of-phase with the sense transcript; its loss results in much stronger phase-shifting in response to light, consistent with an important role in light responses by the clock (Kramer et al. 2003).

\section{Gating of Light Responses}

The circadian clock can modulate its own input; i.e., the response to a standard stimulus is actively modulated depending on time of day through use of an additional clock-associated feedback loop. This process is known as gating and is provided by the vivid ( $v v d)$ gene and VVD protein, which acts to modulate the WC photoreceptor complex. For any photoresponsive cell with just a single photoreceptor having typical reciprocity for duration and intensity (see, e.g., the Neurospora photoreceptor; Crosthwaite et al. 1995), after some minutes in moderate light, the response would be saturated and the cell would be unable to "see" any change in intensity; VVD confers this ability, a process known as photoadaptation (Schwerdtfeger and Linden 2003). Like WC-1, VVD is a member of the PAS protein superfamily (Heintzen et al. 2001) and also a photoreceptor that binds a flavin, in this case an FMN chromophore (Schwerdtfeger and Linden 2003; Zoltowski et al. 2007). When the flavin absorbs blue light, it undergoes a transient covalent addition to the VVD protein, resulting in structural changes that are propagated to the surface of the protein and end with the release of an amino-terminal helix (Zoltowski et al. 2007). This in some way causes VVD to change other proteins, likely kinases, that modulate the activity of the WCC and thereby regulate its activity, although this has not yet been verified biochemically. Significant expression of VVD is restricted to the first day in constant darkness and is controlled in part by the clock (Heintzen et al. 2001), thus accounting for the finding that light signals elicit reduced clock-resetting effects when delivered on the first day after a light-to-dark transfer (Dharmananda 1980). VVD is not required for circadian rhythmicity, but this immediate and transient repressor contributes to circadian entrainment by making dark-to-light transitions more discrete and is important for setting the phase of the overt rhythm. Specifically, VVD allows the dark-to-light transition to be less influential for purposes of phase determination so that the light-to-dark transition at the end of the day determines phase (Elvin et al. 2005), and it also has a major role in ensuring that the phase of the overt rhythm is more or less constant across a range of temperatures (Hunt et al. 2007).

\section{TEMPERATURE EFFECTS ON THE CLOCK}

There are three distinguishable aspects to the response of the circadian system to temperature. First, all clocks, including that of Neurospora, will entrain to temperature steps and cycles, with steps up in temperature resetting the clock in a manner similar to light pulses (see, e.g., Francis and Sargent 1979). Second, there are physiological temperature limits for operation of the clock that lie within the limits for growth. Third, the circadian period length is more or less the same when measured at different constant temperatures, a phenomenon known as "temperature-compensation." Compelling molecular models exist for the first two of these temperature effects as being mediated through the amount and perhaps the form of FRQ protein made, there being no independent temperature sensor (Nowrousian et al. 2003).

\section{Temperature Resetting}

Unlike the case with light, clock resetting by temperature steps is understood in terms of posttranscriptional regulation. Indeed, frq transcript levels are influenced little by temperature, but as described above (Liu et al. 1997), as the temperature increases, so does the level of FRQ. As a result, FRQ levels oscillate around a higher mean at higher temperatures; oscillations with peaks of FRQ in the late day to early evening and troughs in the late night continue, but the number of molecules of FRQ associated with a given "time of day" is different at different temperatures. For instance, Merrow et al. (1997) determined that at peak at $25^{\circ} \mathrm{C}$ there are about 30 molecules of FRQ in the nucleus, fewer than this number at $20^{\circ} \mathrm{C}$ and more at $30^{\circ} \mathrm{C}$ (Fig. 5). Because at the lowest point in the curve (dawn) at $28^{\circ} \mathrm{C}$ (the upper curves in Fig. $5)$ there are more molecules of FRQ per nucleus than at the highest point in the curve (dusk) at $21^{\circ} \mathrm{C}$, a shift in temperature corresponds literally to a step to a different time and a shift in the state of the clock (Liu et al. 1998) even in the absence of any synthesis or turnover of clock components. Following the step, the dynamics of the feedback loop again take control so that the relative levels of $f r q$ and FRQ are adjusted in terms of the new temperature: If there is enough FRQ to shut off WCC, then no more is made and molecular time is around evening, but if more FRQ is needed to complete the feedback and shut off the WCC, then more is made and molecular time is 


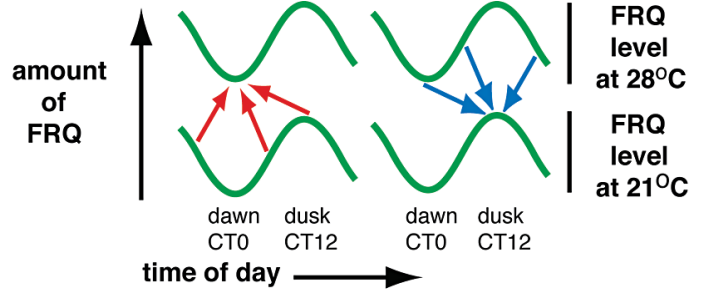

Figure 5. A possible mechanism for how temperature resets the clock. (Green) Cycles in FRQ levels at different temperatures. (Red arrows) Step up at any time leaves the clock with too little FRQ to close the feedback loop, so the clock is reset to the time corresponding to low FRQ, subjective dawn. (Blue arrows) Step down at any time leaves the clock with enough FRQ to close the feedback loop, so the clock is reset to the time corresponding to high FRQ, around subjective evening (see text for details). (Adapted, with permission, from Dunlap 1999 [C Elsevier].)

around dawn. As implicit in this discussion, because temperature acts directly on core components rather than being transduced by a photoreceptor, temperature can be a stronger resetting cue than light (Liu et al. 1998).

\section{Physiological Limits for Rhythmicity}

As noted above, the range of temperatures over which the clock keeps time reflects temperature influences on both the total amount of FRQ and the ratio of the two FRQ forms (Colot et al. 2005; Diernfellner et al. 2005). Temperature-regulated splicing can (at lower temperatures) remove the second intron in frq (see Fig. 2) so that translation initiates at codon 100 of the FRQ open reading frame (ORF). In addition, the absolute level of FRQ protein is controlled by ambient temperature (Liu et al. 1997), in part by the two uORFs in the 5'UTR (untranslated region) of frq (Fig. 2). Strains that can make only one FRQ form show a reduced temperature range permissive for rhythmicity, suggesting that the synthesis of two FRQ forms is a novel adaptive mechanism to extend the physiological temperature range over which the clock can function (Garceau et al. 1997; Liu et al. 1997). Additionally, the clock can function with either form of FRQ exclusively if there is enough of it (Colot et al. 2005; Diernfellner et al. 2005), and there is no difference in stability of the two forms (Liu et al. 1997). It should be noted, however, that the circadian rhythms in strains expressing exclusively either long or short FRQ are clearly temperature-compensated (Fig. 6), contrary to a prior suggestion (Diernfellner et al. 2005). In collaborative work (Diernfellner et al. 2007), we have examined the 1FRQ-only and sFRQ-only strains reported by Diernfellner et al. (2005) and by us (Colot et al. 2005), as well as new strains, and we all agree that the basis of temperature-compensation does not lie in regulation of the relative levels at which the two isoforms of FRQ are made or in properties specific to one of the two isoforms. Instead of a role in compensation, the differential temperature-dependent synthesis of IFRQ and sFRQ may provide a means to fine-tune the period or sculpt the frq mRNA waveform in response to changes in ambient temperature (Diernfellner et al. 2007).

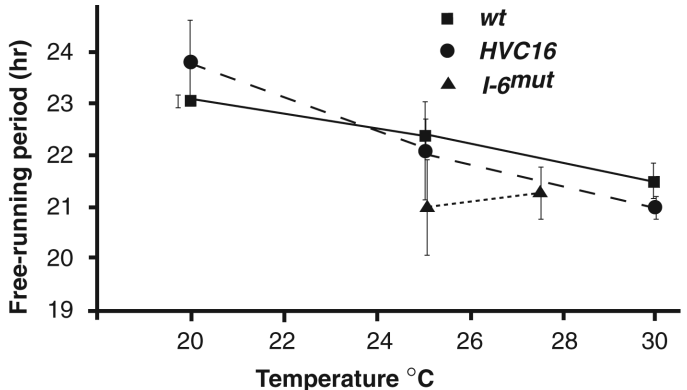

Figure 6. Strains expressing only long FRQ are not deficient in temperature-compensation. Period length is plotted as a function of temperature from a wild-type strain and from two independently engineered strains that express only long FRQ ( $H V C 16$ from Colot et al. 2005; I- $6^{m u t}$ from Diernfellner et al. 2005). The error bars indicate $+/$ - one standard deviation; $n=6$. Both strains clearly show compensation. See text for details.

\section{Temperature-Compensation}

Temperature-compensation is the third way that clocks respond to temperature; it is the last of the canonical circadian properties for which a molecular explanation has not existed, and indeed it has been a difficult problem to study. Precedents show that many period mutants are associated with partial loss of temperature-compensation for (relatively) obvious reasons. For instance, in Drosophila, the dosage of PER influences period length (Smith and Konopka 1982), so mutations that influence the stability of the protein in a temperature-dependent manner will have compensation phenotypes. Likewise, mutations that create an unstable or an unusually stable clock component, either inherent to the protein or secondarily, can have a temperature-compensation phenotype. An example of a secondary effect is the PRD-4 protein in Neurospora.prd-4 is the Neurospora ortholog of the gene encoding the DNA-damage-activated protein checkpoint kinase 2 that can phosphorylate and destabilize FRQ (Pregueiro et al. 2006). A mutation that causes PRD-4 to become active even in the absence of DNA damage results in constant elevated activity of this kinase, resulting in temperature-dependent destabilization of FRQ and a partial loss of temperature-compensation. It is not true, however, that every mutation affecting period necessarily affects compensation; for instance, the short-period mutants $f r q^{1}$ and $f r q^{2}$ are normal in this respect (Feldman et al. 1979).

Because of the likelihood that loss of compensation mutations would not be informative, we instead followed up two distinct mutations that resulted in enhanced compensation, prd-3 and chr. Normally, the Neurospora clock shows a modest undercompensation phenotype whereby the period length gets slightly shorter as the temperature increases up to about $30^{\circ} \mathrm{C}$. Beyond this point, compensation appears to be largely ineffective because the slope of the period versus temperature curve becomes much steeper. In $p r d-3$ and chr, this profile shows overcompensation or extended compensation (Feldman et al. 1979). Both genes were cloned by SNP (single-nucleotide polymorphism) mapping and, surprisingly, they encode separate subunits of the same holoenzyme, casein kinase 2 
(CK2): chr harbors an R265C mutation in the $\beta 1$-subunit tail region of $\mathrm{CK} 2$, which has a role in substrate recognition and is highly conserved among the fungi, and prd-3 harbors a $\mathrm{Y} 43 \mathrm{H}$ mutation in the phosphate anchor region associated with ATP binding near the catalytic core of the $\alpha$ subunit. This circumstantial evidence strongly suggests a role for CK2 in the temperature-compensation process.

Although a role in compensation would be novel, CK2 is, of course, an enzyme with a well-appreciated role in many circadian systems. The $\beta$ subunit CKB3 in Arabidopsis associates with CCA1 and overexpression of CKB3 leads to a shorter period (Sugano et al. 1998). In Neurospora, FRQ has been shown to be phosphorylated in vitro by $\mathrm{CK} 2$ and a partial loss-of-function mutation lengthens period (Yang et al. 2002); finally, in Drosophila, two long-period mutants, Andante and Timekeeper, define CK2 $\beta$ and CK2 $\alpha$, respectively. Thus, CK2 is well positioned in the clock to have a role in compensation, and several approaches have been used to test this possibility. We completed gene disruptions for each subunit to establish definitive nulls and determined that spores lacking CK2 $\alpha$ will not germinate, whereas loss of CK2 $\beta 1(\Delta c k b-1)$ yields viable spores that fail to band rhythmically. This genetic background provides the perfect context in which to assess the role of CK2 dosage in compensation. A transgene was constructed in which the quinic acid-2 (qa-2) promoter was used to drive expression of $c k b-1$ at a level proportional to the amount of the inducer, quinic acid (QA), in the medium; this promoter has previously been extensively used to drive regulated expression in Neurospora (see, e.g., Aronson et al. 1994b; Merrow et al. 1997). In the context of $\Delta c k b-1$ at $25^{\circ} \mathrm{C}$, this $q a-c k b-1$ construct rescues rhythmic banding even with no added inducer due to a very low level of baseline expression. However, the period is quite long under these conditions (28-30 hours), and as the level of CK2 $\beta 1$ is

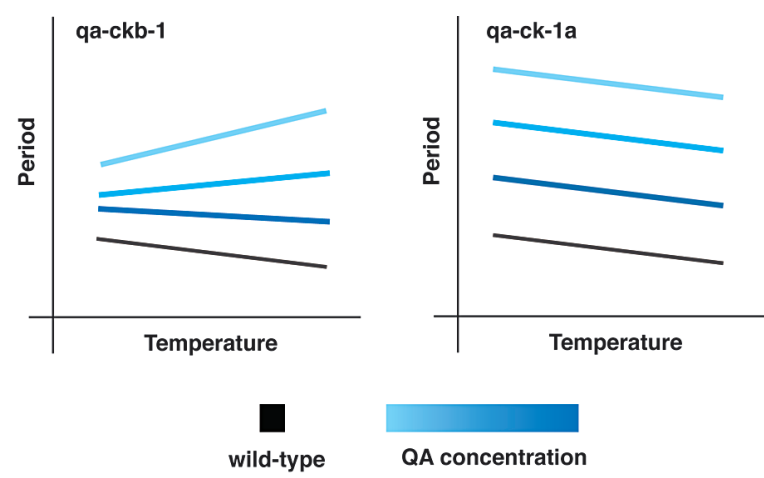

Figure 7. A special role for casein kinase 2 (CK2) in temperature-compensation. Strains bearing inducible copies of the gene encoding the $\beta$ subunit of CK2 (qa-ckb-1) or CK1 (qa-ck1a) were grown at different levels of inducer and at different temperatures. (Left) As levels of $c k b-1$ decrease leading to less CK2 activity, the period length increases and compensation moves from the slight undercompensation typical of wild type to distinct overcompensation. (Right) As CK1 levels drop, the period gets longer, but in contrast to the behavior seen with CK2, the mode of compensation remains like that of wild type. These illustrations depict unpublished data from A. Mehra et al. (in prep.) increased, the period drops steadily to within the wildtype range (Fig. 7, left panel). More interesting is the effect of ambient temperature on this phenomenon. Across a physiological temperature range, strains expressing high levels of CK2 $\beta 1$ are slightly undercompensated in a manner typical for the wild-type clock. As CK2 $\beta 1$ dosage drops, compensation passes through a range of better than normal compensation and as dosage approaches the limit for rhythmic banding, compensation changes to overcompensated, fully mimicking the $c k b$ $I^{c h r}$ overcompensation phenotype. Importantly, however, there are no mutant proteins in these experiments; only the dose of wild-type proteins is being controlled.

These data establish a clear role for CK2 in effecting compensation, but it may be that any mutation in a FRQ kinase will alter compensation. This was tested by performing similar experiments with $\mathrm{CK} 1$, the other principal kinase acting on FRQ. Because CK1 is essential, a knockin strategy was used to replace the normal $c k-1 a$ promoter with the $q a-2$ promoter, thus placing CK1 expression under QA control. As seen in Figure 7 (right panel), reducing the dosage of CK1 has profound effects on period length, serving to increase the period to more than 30 hours. However, and importantly, the temperature-compensation profile was not changed; even the long-period clocks show the mild undercompensation seen for wild type. Similar experiments with consistent results were performed with protein phosphatase 1 (PP1). These data indicate that the role of kinases in compensation is special and that CK2 has such a role. In vitro studies on the activity of the CK2 enzyme have shown that even at low dosage, CK2 displays a normal temperatureactivity profile; i.e., CK2 is more active at higher temperatures, suggesting that it should phosphorylate FRQ to a greater extent. However, and paradoxically, even though CK2 activity increases at higher temperatures, when examined at low CKB-1 levels, FRQ degradation is seen to actually decrease as temperature increases. Because the kinetics of FRQ turnover is a major determinant of period length, these data are consistent with the observation of increasing period as a function of temperature (i.e., overcompensation) at low CK2 levels; moreover, these results recapitulate the original chr phenotype of reduced FRQ turnover and longer period at higher temperatures.

These data are at once surprising and not easy to reconcile with a simple notion of compensation as a result of kinase action. A variety of data beginning with those of Liu et al. (2000) have shown and reconfirmed a central role for FRQ phosphorylation in determining its turnover and of FRQ turnover in determining period length. The simple interpretation of these data would be that higher temperature must yield more kinase activity (as we have confirmed), which ought to yield more rapid turnover and a shorter period. This is seen at high kinase activity; however, at low ckb-1 levels, the reverse is seen: Turnover actually decreases and period length increases at higher temperatures despite greater activity of CK2. Although a complete mechanistic explanation for this is lacking, it remains true that this paradoxical behavior reflects exactly the behavior needed to bring about compensation, namely, a process that acts to decrease the pace of the 
clock at higher temperatures and that normally counters the drive of CK2 for turnover. The focus of these competing activities is FRQ, whose turnover kinetics dictates period length. One possible way to rationalize these results would be the existence of more than one "class" of CK2-influenced phosphorylation sites on FRQ, one class that simply leads to turnover (and longer periods at lower CK2 doses) and another "class" whose phosphorylation might be competed by an unknown additional activity. In this scenario, compensation is achieved by the balance of these activities, and at low doses of CK2, the competing activity wins out, leading to overcompensation. A prediction that follows is that mutation of one or more of the phosphorylation sites in the second class would lead to an extended or overcompensation phenotype like that seen in $c k b-1^{c h r}$. We are now testing this hypothesis.

\section{ACKNOWLEDGMENTS}

This work was supported by grants from the National Institutes of Health to J.C.D. (GM34985 and GM068087) and to J.C.D. and J.J.L. (GM083336). L.F.L. is a Pew Latin American Fellow.

\section{REFERENCES}

Allada R., White N.E., So W.V., Hall J.C., and Rosbash M. 1998. A mutant Drosophila homolog of mammalian CLOCK disrupts circadian rhythms and transcription of period and timeless. Cell 93: 805 .

Antoch M., Soog E., Chang A., Vitaterna M., Zhao Y., Wilsbacher L., Sangoram A., King D., Pinto L., and Takahashi J. 1997. Functional identification of the mouse circadian CLOCK gene by transgenic BAC rescue. Cell 89: 655.

Aronson B.D., Johnson K.A., and Dunlap J.C. 1994a. The circadian clock locus frequency: A single ORF defines period length and temperature compensation. Proc. Natl. Acad. Sci. 91: 7683

Aronson B., Johnson K., Loros J.J., and Dunlap J.C. 1994b. Negative feedback defining a circadian clock: Autoregulation in the clock gene frequency. Science 263: 1578 .

Ballario P. and Macino G. 1997. White collar proteins: PASsing the light signal in Neurospora crassa. Trends Microbiol. 5: 458.

Ballario P., Talora C., Galli D., Linden H., and Macino G. 1998. Roles in dimerization and blue light photoresponse of the PAS and LOV domains of Neurospora crassa WHITE COLLAR proteins. Mol. Microbiol. 29: 719.

Ballario P., Vittorioso P., Magrelli A., Talora C., Cabibbo A., and Macino G. 1996. White collar-1, a central regulator of blue-light responses in Neurospora crassa, is a zinc-finger protein. EMBO J. 15: 1650.

Belden W.J., Loros J.J., and Dunlap J.C. 2007a. Execution of the circadian negative feedback loop in Neurospora requires the ATP-dependent chromatin-remodeling enzyme CLOCKSWITCH. Mol. Cell 25: 587.

Belden W.J., Larrondo L.F., Froehlich A.C., Shi M., Chen C.-H., Loros J.J., and Dunlap J.C. 2007b. The band mutation in Neurospora crassa is a dominant allele of ras- 1 implicating RAS-signaling in circadian output. Genes Dev. 21: 1494.

Bell-Pedersen D., Cassone V.M., Earnest D.J., Golden S.S., Hardin P.E., Thomas T.L., and Zoran M.J. 2005. Circadian rhythms from multiple oscillators: Lessons from diverse organisms. Nat. Rev. Genet. 6: 544.

Bünning E. 1973. The physiological clock. Springer-Verlag, New York

Cheng P., He Q., He Q., Wang L., and Liu Y. 2005. Regulation of the Neurospora circadian clock by an RNA helicase. Genes Dev. 19: 234 .
Collett M.A., Garceau N., Dunlap J.C., and Loros J.J. 2002. Light and clock expression of the Neurospora clock gene frequency is differentially driven by but dependent on WHITE COLLAR-2. Genetics 160: 149.

Colot H.V., Loros J.J., and Dunlap J.C. 2005. Temperature-modulated alternative splicing and promoter use in the circadian clock gene frequency. Mol. Biol. Cell 16: 5563.

Colot H.V., Park G., Turner G.E., Ringelberg C., Crew C.M., Litvinkova L., Weiss R.L., Borkovich K.A., and Dunlap J.C. 2006. A high-throughput gene knockout procedure for Neurospora reveals functions for multiple transcription factors. Proc. Natl. Acad. Sci. 103: 10352.

Crosson S. and Moffat K. 2001. Structure of a flavin-binding plant photoreceptor domain: Insights into light mediated signal transduction. Proc. Natl. Acad. Sci. 98: 2995.

Crosthwaite S.C., Dunlap J.C., and Loros J.J. 1997. Neurospora $w c-1$ and $w c-2$ : Transcription, photoresponses, and the origins of circadian rhythmicity. Science 276: 763.

Crosthwaite S.C., Loros J.J., and Dunlap J.C. 1995. Lightinduced resetting of a circadian clock is mediated by a rapid increase in frequency transcript. Cell 81: 1003.

Darlington T.K., Wager-Smith K., Ceriani M.F., Staknis D., Gekakis N., Steeves T., Weitz C.J., Takahashi J.S., and Kay S.A. 1998. Closing the circadian loop: CLOCK induced transcription of its own inhibitors, per and tim. Science 280: 1599.

Denault D.L., Loros J.J., and Dunlap J.C. 2001. WC-2 mediates WC-1-FRQ interaction within the PAS protein-linked circadian feedback loop of Neurospora crassa. EMBO J. 20: 109.

dePaula R.M., Lewis Z.A., Greene A.V., Seo K.S., Morgan L.W., Vitalini M.W., Bennett L., Gomer R.H., and BellPedersen D. 2006. Two circadian timing circuits in Neurospora crassa share components and regulate distinct rhythmic processes. J. Biol. Rhythms 21: 159.

Dharmananda S. 1980. "Studies of the circadian clock of Neurospora crassa: Light-induced phase shifting." Ph.D. thesis, University of California, Santa Cruz.

Diernfellner A.C., Schafmeier T., Merrow M.W., and Brunner M. 2005. Molecular mechanism of temperature sensing by the circadian clock of Neurospora crassa. Genes Dev. 19: 1968.

Diernfellner A., Colot H.V., Dintsis O., Loros J.J., Dunlap J.C., and Brunner M. 2007. Long and short isoforms of Neurospora clock protein FRQ support temperature compensated circadian rhythms. FEBS Lett. (in press).

Dunlap J.C. 1999. Molecular bases for circadian clocks. Cell 96: 271.

. 2006. Proteins in the Neurospora circadian clockworks. J. Biol. Chem. 281: 28489.

Dunlap J.C. and Loros J.J. 2006. How fungi keep time: Circadian system in Neurospora and other fungi. Curr. Opin. Microbiol. 9: 579.

Elvin M., Loros J.J., Dunlap J.C., and Heintzen C. 2005. The PAS/LOV protein VIVID supports a rapidly dampened daytime oscillator that facilitates entrainment of the Neurospora circadian clock. Genes Dev. 19: 2593.

Feldman J.F., Gardner G.F., and Dennison R.A. 1979. Genetic analysis of the circadian clock of Neurospora. In Biological rhythms and their central mechanism (ed. M. Suda), p. 57. Elsevier, Amsterdam.

Francis C. and Sargent M.L. 1979. Effects of temperature perturbations on circadian conidiation in Neurospora. Plant Physiol. 64: 1000

Froehlich A.C. 2002. "Light and circadian regulation in Neurospora crassa." Ph.D. thesis, Dartmouth College, Hanover, New Hampshire.

Froehlich A.C., Loros J.J., and Dunlap J.C. 2002. WHITE COLLAR-1, a circadian blue light photoreceptor, binding to the frequency promoter. Science 297: 815 .

. 2003. Rhythmic binding of a WHITE COLLAR containing complex to the frequency promoter is inhibited by FREQUENCY. Proc. Natl. Acad. Sci. 100: 5914

Froehlich A.C., Noh B., Vierstra R.D., Loros J., and Dunlap J.C. 2005. Genetic and molecular analysis of phytochromes from the filamentous fungus Neurospora crassa. Eukaryot. Cell 4: 2140. Garceau N. 1996. "Molecular and genetic studies on the frq and 
ccg-1 loci of Neurospora." Ph.D. thesis, Dartmouth College, Hanover, New Hampshire.

Garceau N., Liu Y., Loros J.J., and Dunlap J.C. 1997. Alternative initiation of translation and time-specific phosphorylation yield multiple forms of the essential clock protein FREQUENCY. Cell 89: 469.

Gorl M., Merrow M., Huttner B., Johnson J., Roenneberg T., and Brunner M. 2001. A PEST-like element in FREQUENCY determines the length of the circadian period in Neurospora crassa. EMBO J. 20: 7074

Harding R.W. and Shropshire W.J. 1980. Photocontrol of carotenoid biosynthesis. Annu. Rev. Plant Physiol. 31: 217.

He Q. and Liu Y. 2005. Molecular mechanism of light responses in Neurospora: From light-induced transcription to photoadaptation. Genes Dev. 19: 2888

He Q., Cheng P., Yang Y., He Q., Yu Q., and Liu Y. 2003. FWD1 mediated degradation of FREQUENCY in Neurospora establishes a conserved mechanism for circadian clock regulation. $E M B O$ J. 22: 4421 .

He Q., Cheng P., Yang Y., Wang L., Gardner K., and Liu Y. 2002. WHITE COLLAR-1, a DNA binding transcription factor and a light sensor. Science 297: 840

Heckman D.S., Geiser D.M., Eidell B.R., Stauffer R.L., Kardos N.L., and Hedges S.B. 2001. Molecular evidence for the early colonization of land by fungi and plants. Science 293: 1129 .

Heintzen C. and Liu Y. 2007. The Neurospora crassa circadian clock. Adv. Genet. 58: 25

Heintzen C., Loros J.J., and Dunlap J.C. 2001. VIVID, gating and the circadian clock: The PAS protein VVD defines a feedback loop that represses light input pathways and regulates clock resetting. Cell 104: 453

Hunt S.M., Elvin M., Crosthwaite S.K., and Heintzen C. 2007. The PAS/LOV protein VIVID controls temperature compensation of circadian clock phase and development in Neurospora crassa. Genes Dev. 21: 1964.

Hunter-Ensor M., Ousley A., and Sehgal A. 1996. Regulation of the Drosophila protein TIMELESS suggests a mechanism for resetting the circadian clock by light. Cell 84: 677 .

Ingold C.T. 1971. Fungal spores. Clarendon Press, Oxford, United Kingdom.

Ko H.W., Jiang J., and Edery I. 2002. Role for Slimb in the degradation of Drosophila Period protein phosphorylated by Doubletime. Nature 420: 673

Kramer C., Loros J.J., Dunlap J.C., and Crosthwaite S.K. 2003. Role for antisense RNA in regulating circadian clock function in Neurospora crassa. Nature 421: 948.

LaCava J., Houseley J., Saveanu C., Petfalski E., Thompson E., Jacquier A., and Tollervey D. 2005. RNA degradation by the exosome is promoted by a nuclear polyadenylation complex. Cell 121: 713

Lee K., Loros J.J., and Dunlap J.C. 2000. Interconnected feedback loops in the Neurospora circadian system. Science 289: 107.

Linden H. and Macino G. 1997. White collar-2, a partner in bluelight signal transduction, controlling expression of light-regulated genes in Neurospora crassa. EMBO J. 16: 98.

Liu Y. Loros J., and Dunlap J.C. 2000. Phosphorylation of the Neurospora clock protein FREQUENCY determines its degradation rate and strongly influences the period length of the circadian clock. Proc. Natl. Acad. Sci. 97: 234.

Liu Y., Garceau N., Loros J.J., and Dunlap J.C. 1997. Thermally regulated translational control mediates an aspect of temperature compensation in the Neurospora circadian clock. Cell 89: 477.

Liu Y., Merrow M., Loros J.J., and Dunlap J.C. 1998. How temperature changes reset a circadian oscillator. Science 281: 825 .

Loros J.J. and Feldman J.F. 1986. Loss of temperature compensation of circadian period length in the frq-9 mutant of Neurospora crassa. J. Biol. Rhythms 1: 187.

Loros J.J., Denome S.A., and Dunlap J.C. 1989. Molecular cloning of genes under the control of the circadian clock in Neurospora. Science 243: 385.

Luo C., Loros J.J., and Dunlap J.C. 1998. Nuclear localization is required for function of the essential clock protein FREQUENCY. EMBO J. 17: 1228

McClung C.R., Fox B.A., and Dunlap J.C. 1989. The Neurospora clock gene frequency shares a sequence element with the Drosophila clock gene period. Nature 339: 558.

Merrow M., Garceau N., and Dunlap J.C. 1997. Dissection of a circadian oscillation into discrete domains. Proc. Natl. Acad. Sci. 94: 3877.

Merrow M., Franchi L., Dragovic Z., Gorl M., Johnson J., Brunner M., Macino G., and Roenneberg T. 2001. Circadian regulation of the light input pathway in Neurospora crassa. EMBO J. 20: 307

Nakajima M., Imai K., Ito H., Nishiwaki T., Murayama Y., Iwasaki H., Oyama T., and Kondo T. 2005. Reconstitution of circadian oscillation of cyanobacterial KaiC phosphorylation in vitro. Science 308: 414

Nowrousian M., Duffield G.E., Loros J.J., and Dunlap J.C. 2003. The frequency gene is required for temperature-dependent regulation of many clock-controlled genes in Neurospora crassa. Genetics 164: 922.

Pittendrigh C.S., Bruce V.G., Rosenzweig N.S., and Rubin M.L. 1959. A biological clock in Neurospora. Nature 184: 169.

Pregueiro A., Liu Q., Baker C., Dunlap J.C., and Loros J.J. 2006. The Neurospora checkpoint kinase 2: A regulatory link between the circadian and cell cycles. Science 313: 644 .

Ruoff P., Loros J.J., and Dunlap J.C. 2005. The relationship between FRQ-protein stability and temperature compensation in the Neurospora circadian clock. Proc. Natl. Acad. Sci. 102: 17681.

Rutila J.E., Suri V., Le M., So W.V., Rosbash M., and Hall J.C. 1998. CYCLE is a second bHLH-PAS clock protein essential for circadian rhythmicity and transcription of Drosophila period and timeless. Cell 93: 805 .

Sargent M.L. and Briggs W.R. 1967. The effect of light on a circadian rhythm of conidiation in Neurospora. Plant Physiol. 42: 1504

Sargent M.L. and Kaltenborn S.H. 1972. Effects of medium composition and carbon dioxide on circadian conidiation in Neurospora. Plant Physiol. 50: 171

Sargent M.L., Briggs W.R., and Woodward D.O. 1966. The circadian nature of a rhythm expressed by an invertaseless strain of Neurospora crassa. Plant Physiol. 41: 1343.

Schafmeier T., Kaldi K., Diernfellner A., Mohr C., and Brunner M. 2006. Phosphorylation-dependent maturation of Neurospora circadian clock protein from a nuclear repressor toward a cytoplasmic activator. Genes Dev. 20: 297.

Schafmeier T., Haase A., Kaldi K., Scholz J., Fuchs M., and Brunner M. 2005. Transcriptional feedback of Neurospora circadian clock gene by phosphorylation-dependent inactivation of its transcription factor. Cell 122: 235 .

Schwerdtfeger C. and Linden H. 2003. VIVID is a flavoprotein and serves as a fungal blue light photoreceptor for photoadaptation. EMBO J. 22: 4846

Shigeyoshi Y., Taguchi K., Yamamoto S., Takeida S., Yan L., Tei H., Moriya S., Shibata S., Loros J.J., Dunlap J.C., and Okamura H. 1997. Light-induced resetting of a mammalian circadian clock is associated with rapid induction of the mPerl transcript. Cell 91: 1043

Smith R.F. and Konopka R.J. 1982. Effects of dosage alterations at the per locus on the period of the circadian clock of Drosophila. Mol. Gen. Genet. 185: 30.

Sugano S., Andronis C., Green R., Wang Z., and Tobin E. 1998. Protein kinase CK2 interacts with and phosphorylates the Arabidopsis circadian clock-associated gene 1 protein. Proc. Natl. Acad. Sci. 95: 11020.

Sweeney B.M. 1976. Circadian rhythms, definition and general characterization. In The molecular basis of circadian rhythms (report of the Dahlem Workshop) (ed. J.W. Hastings and H.G. Schweiger), p. 77. Abakon-Verlagsgesellschaft, Berlin.

Tan Y., Dragovic Z., Merrow M., and Roenneberg T. 2004. Entrainment dissociates transcription and translation of a circadian clock gene in Neurospora. Curr. Biol. 14: 433.

Yang Y., Cheng P., and Liu Y. 2002. Regulation of the Neurospora circadian clock by casein kinase II. Genes Dev. 16: 994.

Zoltowski B.D., Schwerdtfeger C., Widom J., Loros J.J., Bilwes A.M., Dunlap J.C., and Crane B.R. 2007. Conformational switching in the fungal light sensor Vivid. Science 316: 1054 


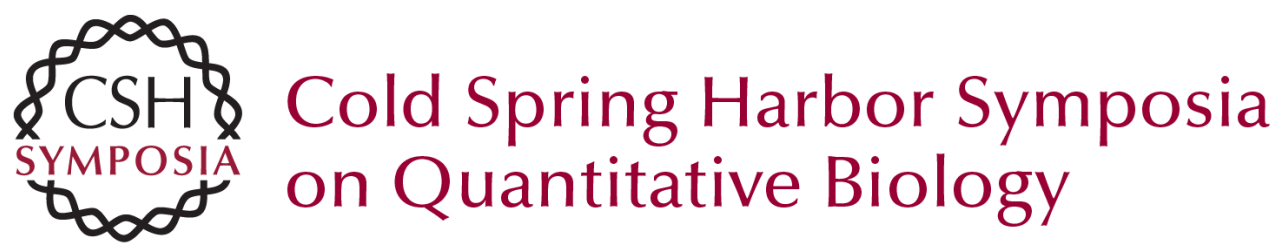

\section{A Circadian Clock in Neurospora: How Genes and Proteins Cooperate to Produce a Sustained, Entrainable, and Compensated Biological Oscillator with a Period of about a Day}

J.C. Dunlap, J.J. Loros, H.V. Colot, et al.

Cold Spring Harb Symp Quant Biol 2007 72: 57-68

Access the most recent version at doi:10.1101/sqb.2007.72.072

References This article cites 72 articles, 43 of which can be accessed free at:

http://symposium.cshlp.org/content/72/57.full.html\#ref-list-1

License

Email Alerting Receive free email alerts when new articles cite this article - sign up in the box at the

Service top right corner of the article or click here. 